\title{
Adaptability of the nociceptive withdrawal reflex [version 1;
}

\section{peer review: 2 approved]}

\author{
Nathan Eckert ${ }^{1}$, Zachary A Riley ${ }^{1,2}$ \\ ${ }^{1}$ Department of Kinesiology \& Program in Neural Science, Indiana University, Bloomington IN, 47405, USA \\ ${ }^{2}$ Department of Kinesiology, Indiana University-Purdue University Indianapolis, Indianapolis IN, 46202, USA
}

V1 First published: 16 Jul 2013, 2:158
https://doi.org/10.12688/f1000research.2-158.v1

Latest published: 19 Dec 2013, 2:158

https://doi.org/10.12688/f1000research.2-158.v2

\begin{abstract}
The nociceptive withdrawal reflex is a protective mechanism to mediate interactions within a potentially dangerous environment. The reflex is formed by action-based sensory encoding during the early post-natal developmental period, and it is unknown if the protective motor function of the nociceptive withdrawal reflex in the human upper-limb is adaptable based on the configuration of the arm or if it can be modified by short-term practice of a similar or opposing motor action. In the present study, nociceptive withdrawal reflexes were evoked by a brief train of electrical stimuli applied to digit II, 1) in five different static arm positions and, 2) before and after motor practice that was opposite (EXT) or similar (FLEX) to the stereotyped withdrawal response, in 10 individuals. Withdrawal responses were quantified by the electromyography (EMG) reflex response in several upper limb muscles, and by the forces and moments recorded at the wrist. EMG onset latencies and response amplitudes were not significantly different across the arm positions or between the EXT and FLEX practice conditions, and the general direction of the withdrawal response was similar across arm positions. In addition, the force vectors were not different after practice in either the practice condition or between EXT and FLEX conditions. We conclude the withdrawal response is insensitive to changes in elbow or shoulder joint angles as well as remaining resistant to short-term adaptations from the practice of motor actions, resulting in a generalized limb withdrawal in each case. It is further hypothesized that the multisensory feedback is weighted differently in each arm position, but integrated to achieve a similar withdrawal response to safeguard against erroneous motor responses that could cause further harm. The results remain consistent with the concept that nociceptive withdrawal reflexes are shaped through long-term and not short-term action based sensory encoding.
\end{abstract}

Keywords

reflex, nociception, withdrawal response, electromyography, sensory encoding

\section{Open Peer Review \\ Approval Status \\ 1 \\ 2 \\ version 2 \\ (revision) \\ 19 Dec 2013 \\ version 1 \\ 16 Jul 2013

$\checkmark$

view
view \\ 1. Hong-You Ge, Aalborg University, Aalborg East, Denmark \\ 2. Brach Poston, Cleveland Clinic Lerner Research Institute, Cleveland, OH, USA Any reports and responses or comments on the article can be found at the end of the article.}


Corresponding author: Zachary A Riley (zachary.riley@gmail.com)

Competing interests: No competing interests were disclosed.

Grant information: The author(s) declared that no grants were involved in supporting this work.

Copyright: @ 2013 Eckert $\mathrm{N}$ and Riley ZA. This is an open access article distributed under the terms of the Creative Commons Attribution License, which permits unrestricted use, distribution, and reproduction in any medium, provided the original work is properly cited. Data associated with the article are available under the terms of the Creative Commons Zero "No rights reserved" data waiver (CC0 1.0 Public domain dedication).

How to cite this article: Eckert $\mathrm{N}$ and Riley ZA. Adaptability of the nociceptive withdrawal reflex [version 1; peer review: 2 approved] F1000Research 2013, 2:158 https://doi.org/10.12688/f1000research.2-158.v1

First published: 16 Jul 2013, 2:158 https://doi.org/10.12688/f1000research.2-158.v1 


\section{Introduction}

Noxious electrical stimulation of the digits in humans produces a coordinated reflex response that results in the withdrawal of the limb, akin to removing the hand from touching a hot stove $e^{1,2}$. This reflexive action, known as the nociceptive withdrawal reflex $^{3}$, is an essential protective mechanism for interactions between parts of the body and the environment, allowing for quick removal from noxious stimuli. The relatively short-latency reflex responses occur as a result of transmission from $A \delta$ afferent fibers to the motor neuron pools of several muscles 4 . Previously, it was suggested that stimulation of these afferent fibers consistently produces excitatory postsynaptic potentials in flexor motor neurons and inhibitory postsynaptic potentials in extensor motor neurons of the cat hindlimb ${ }^{5}$. However, fixed nociceptive input would limit the protective capabilities of the nervous system to only producing flexion at individual joints (e.g. elbow, shoulder), and a summary of more recent work has dispelled this hypothesis (see review by Clarke and Harris $\left.(2004)^{6}\right)$.

To initiate reflexive motor actions such as withdrawing the whole limb, nociceptive afferent input would need to be distributed to the motor neuron pools of muscles across several joints to coordinate the movement. In the hindlimb of rats activation of afferent neurons with receptive fields in a specific area of skin coordinates the activity of one or more muscles best suited to remove it from the noxious stimuli ${ }^{3,7}$. Specifically, the pairing of afferent neurons and resulting muscle activity was designated as reflex "modules"3,7,8. Reflex modules are not limited to only activating the synergist muscles required to withdraw the limb, but can also inhibit muscles that would oppose it. Thus, organizing nociceptive sensory-motor interactions into reflex modules would result in a more efficient, coordinated limb withdrawal.

It has been suggested that the reflex modules are shaped by use, or action-based sensory encoding ${ }^{9,10}$. As a purely protective mechanism, the nociceptive withdrawal reflex adapts or develops according to the repetitive motor actions performed and the environment in which there are constant sensory-motor interactions. Since most of these adaptations transpire early in post-natal development ${ }^{11-15}$ it is assumed that they shape the functional sensory-motor behaviors required later in maturity ${ }^{14}$. However, it is unknown how reflex modules are influenced by short-term action-based motor practice or use after development has ceased. Features such as habituation and dishabituation (wind-up), as well as human correlates of long-term potentiation (LTP) and long-term depression (LTD), have been well described in relation to short-term synaptic plasticity in nociceptive pathways $^{2,16-19}$. However, it is yet to be determined how shortterm synaptic plasticity of nociceptive pathways controls the activation of reflex modules translating to the appropriate motor actions. Furthermore, the evidence presented for the activation of specific reflex modules has been demonstrated only by stimulating different receptive fields ${ }^{3,7}$.

The purpose of the present study is to test the adaptability of the nociceptive withdrawal reflex through two different tasks. First, the study seeks to determine if different nociceptive reflex modules are activated in the upper-limb of unimpaired humans by stimulating the same receptive field with the arm in different static arm positions by independently changing either the elbow or shoulder joint angles. We hypothesized that the static muscle length changes would alter group II afferent input that has been shown to have a strong excitatory input to interneurons regulating the withdrawal response ${ }^{20-22}$. Specifically, if there was modular organization in the spinal cord, we expected to observe different patterns of muscle activity (latencies, amplitude), and subsequent endpoint forces, evoked by stimulation in the various positions. Secondly, the study seeks to determine whether the protective motor function of the nociceptive withdrawal reflex in the upper-limb could be modified by short-term practice of a similar or opposing motor action voluntarily triggered by a non-noxious stimuli.

\section{Experimental procedure}

\section{Participants}

Ten healthy adults ( 7 males: $29.9 \pm 7.5$ yrs; range: $21-42$ yrs) participated in the study. None of the subjects reported any neurological disorders or other upper limb musculoskeletal impairments. Each subject provided informed consent prior to participating. The protocol was approved by the Indiana University Institutional Review Board (Study \# 1105005484) and was performed in accordance with the Declaration of Helsinki.

\section{Joint position task}

Nociceptive withdrawal reflexes were examined in five static conditions (see Figure 1A-C) where the arm was fully supported in front of the body in a general position similar to reaching to a stove top or opening a cabinet door. The five conditions were achieved by independently manipulating the elbow or shoulder joint angles in the transverse plane:

1) With the elbow flexed and the shoulder in a neutral position $\left(\mathrm{ELB}_{\mathrm{FLEX}} ; \theta=75^{\circ}\right.$ and $95^{\circ}$, respectively);

2) Elbow extended and shoulder neutral $\left(\mathrm{ELB}_{\mathrm{EXT}} ; \theta=115^{\circ}\right.$ and $95^{\circ}$, respectively);

3) Elbow and shoulder both neutral (NEUT; $\theta=95^{\circ}$ for both);

4) Shoulder flexed and elbow neutral $\left(\mathrm{SHL}_{\mathrm{FLEX}} ; \theta=80^{\circ}\right.$ and $95^{\circ}$, respectively);

5) Shoulder extended and elbow neutral $\left(\mathrm{SHL}_{\mathrm{EXT}} ; \theta=120^{\circ}\right.$ and $95^{\circ}$, respectively). 

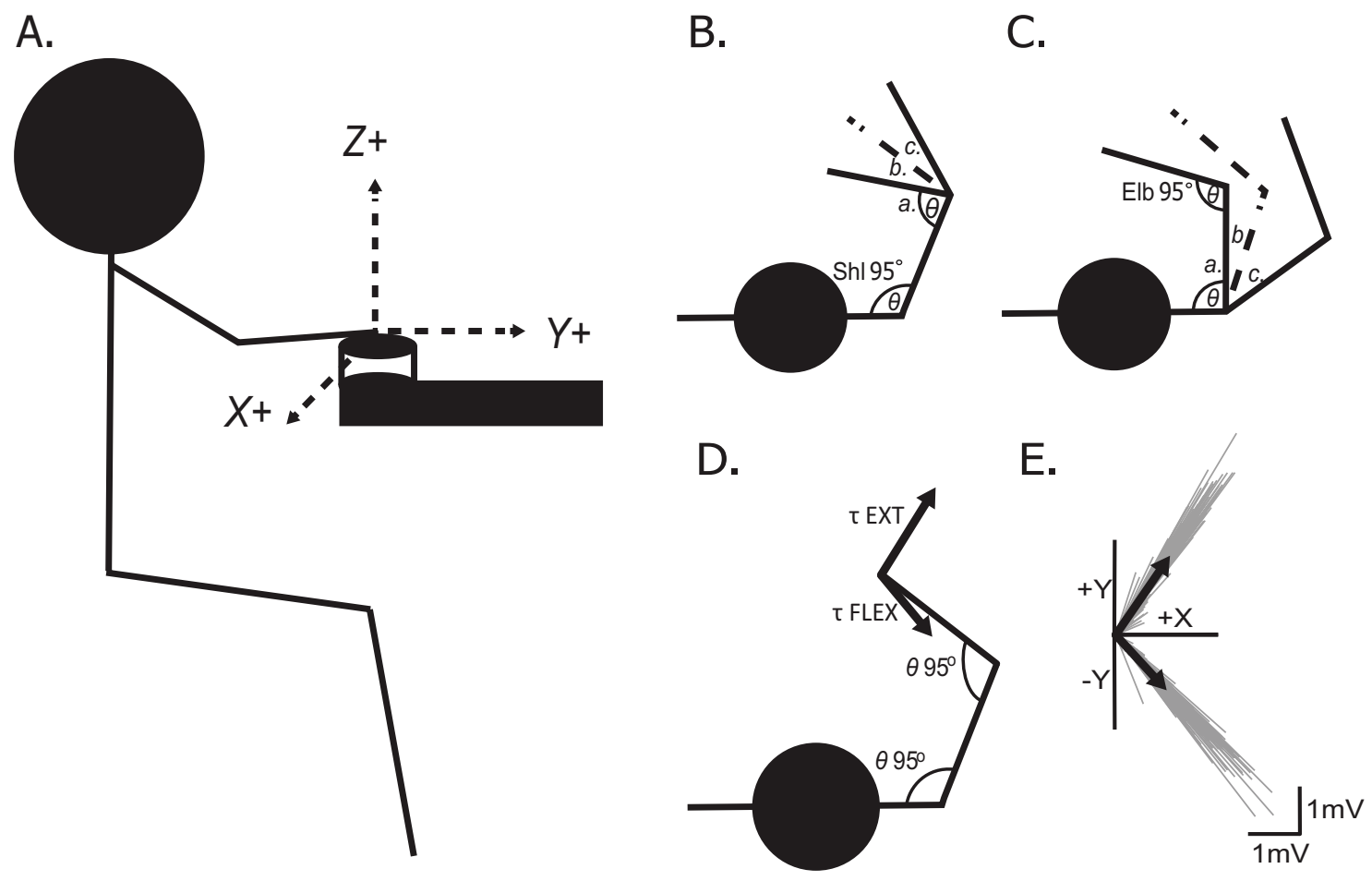

Figure 1. Schematic showing the joint configurations and details of the practice task. A) Sagittal view of the subject with the wrist secured to the force transducer and the orientation of the forces recorded for all of the experiments. B) When independently changing elbow angle (a. ELBFLEX; b. NEUT; c. ELBEXT) while keeping the same shoulder angle $\left(\theta=95^{\circ}\right)$; and $\mathbf{C}$ ) When independently changing shoulder angle (a. SHLFLEX; b. NEUT; c. SHLEXT) while keeping the same elbow angle $\left(\theta=95^{\circ}\right)$. Note that the dashed line (b.) in both $\mathrm{B}$ and $\mathrm{C}$ is the same NEUT condition and was only tested once during the trials and also again after all of the joint positions had been completed. D) Schematic showing the joint configuration ( $\tau=$ elbow $\sim 95^{\circ}$, shoulder $\sim 95^{\circ}$ ) for both EXT and FLEX practice protocols. E) Representative data from one subject demonstrating the magnitude and direction of the endpoint force vector from the nociceptive withdrawal response, after practice, from the individual trials (gray-thin lines) and the average responses (black-thick lines) in the (+/-) Y and $+X$ directions. The average responses from the EXT and FLEX trials are also superimposed on panel D.

When the subject was fixed in each position shoulder abduction was $-75^{\circ}$ and the only attachment point was at the wrist where it was affixed to the force transducer. This allowed the subject to completely relax the limb and did not require any stabilizing muscle activation from the shoulder or surrounding muscles. Each subject completed the five static conditions in a single experimental session, though the order of conditions was randomized for each subject. To examine if there was habituation in the withdrawal response the NEUT condition was repeated at the end of every experiment.

\section{Conditioning task}

Nociceptive withdrawal reflexes were also examined in the right upper-limb of the subjects before and after two practice conditions performed on separate days. In this task the subject was in the aforementioned NEUT position for all of the trials. For the extension practice condition (EXT) the subject was asked to produce a submaximal, voluntary extension of their arm, as quickly as possible, against the force transducer upon receiving non-painful electrical stimulation of digit II (index finger). As the subject was secured at the wrist, this was an isometric contraction. This was repeated for 200 trials. The conditioning stimulations were delivered randomly with a period of $5-15 \mathrm{~s}$ between stimuli in order to eliminate any fatiguing influence. The second condition, flexion practice (FLEX), was performed on a different day and required the subject to isometrically flex the arm at the elbow as quickly as possible. The experimental setup and endpoint force vectors generated by one subject in each practice condition are displayed in Figure 1D\&E. Nociceptive withdrawal reflexes were assessed before and after the completion of the 200 trials in each condition. Shoulder and elbow flexion were kept at a constant $-95^{\circ}$ and shoulder abduction was maintained at $-75^{\circ}$ during each of the testing sessions. There was a minimum of two weeks between testing the two conditions and the order in which they were performed was randomized.

For all testing the subjects were comfortably seated in an upright position with only the right wrist secured to a six degree-of-freedom force transducer (75E20; JR-3, Woodland, CA), which kept them in a static position with each arm configuration. The JR-3 force transducer continuously sampled 
endpoint forces ( $\mathrm{Fx}, \mathrm{Fy}, \mathrm{Fz})$ and moments $(\mathrm{Mx}, \mathrm{My}, \mathrm{Mz})$ during the experiments at $200 \mathrm{~Hz}$. There were constant fluctuations in the gravitational force and moment $(\mathrm{Fz}, \mathrm{Mz})$ with the position of the limb in the two tasks, so this axis of force was excluded from further analysis.

\section{Stimulation conditions}

For both the joint position task and the conditioning task noxious stimulation was applied using a Grass S88X stimulator (Grass Technologies, Astro-Med, USA) connected in-series with a Digitimer DS7AH constant current electrical stimulator (Digitimer LTD, UK). Short trains of electrical stimuli were delivered to digit II using stainless steel ring electrodes secured to the medial and proximal phalanges, while the subject was at rest. Each stimulus train consisted of 10 pulses $(200 \mu \mathrm{s} \mathrm{du}$ ration) delivered at $300 \mathrm{~Hz}$, which was consistent with previous research evoking withdrawal reflexes in the upper limb at rest ${ }^{2}$. Electrical current from the stimulator was slowly increased to determine perceptual threshold at the beginning of the experiment. Then it was increased in $-2 \mathrm{~mA}$ steps (relative to perceptual threshold) until the subject reported that further increases were intolerable. This resulted in a stimulus intensity of between $30-50 \mathrm{~mA}$ for all subjects, which was consistent with other studies in the upper limb $b^{1,23,24}$. The stimulus trains used to elicit the reflex response during the experimental protocols were delivered at random intervals between 5 and $15 \mathrm{~s}$. Eight stimulus trains were delivered in all experiments where the nociceptive withdrawal reflex was examined. Stimulation intensity remained constant across all conditions for a given subject within each day of testing.

In the conditioning task non-noxious stimulation for the 200 practice trials was performed at an intensity of 5-15 mA (1.5× perceptual threshold). The same stimulation trains were delivered during the practice trials, only at a much lower intensity that was not sufficient to evoke a noticeable short-latency reflex in any of the muscles. Stimulus trains were delivered randomly between 5 and $15 \mathrm{~s}$ for the practice trials as well. Five minutes of rest was given between the initial nociceptive withdrawal reflex testing and the practice trials, and between the practice trials and nociceptive withdrawal post-testing.

\section{Electromyography}

Surface electromyography (EMG) signals were recorded the same in both tasks, with single differential bar electrodes (Delsys Inc, MA, USA). The signals were amplified and conditioned using a 16-channel Bagnoli EMG System (Delsys) with high- and low-pass cut-off frequencies of $20 \mathrm{~Hz}$ and $1,000 \mathrm{~Hz}$, respectively, before being stored at a final gain of $1,000 \mathrm{~Hz}$ with Spike2 software (CED, Cambridge, UK). Surface EMG activity was recorded from the abductor pollicus brevis (APB), brachioradialis (BRD), biceps brachii long head (BBL), triceps brachii lateral head (TRI), anterior deltoid (AD), and posterior deltoid (PD) muscles in the right upper limb. The right (ESR) and left erector spinae (ESL) were also recorded to monitor postural responses. The skin overlying each muscle was cleaned prior to affixing the electrode over the individual muscle belly. A single reference electrode was placed over the acromion process.

\section{Data analysis}

Forces and moments were considered relative to subject coordinates with the $+\mathrm{X}$ direction pointing to the right of the subject, the $-Y$ direction pointing towards the subject, and the $+Z$ direction pointing upwards. Analysis of the endpoint forces and moments were confined to the horizontal (X-Y) plane. For all of the nociceptive withdrawal reflex trials the resultant twodimensional $(\mathrm{X}-\mathrm{Y})$ endpoint force vectors were computed from the local peak force up to $200 \mathrm{~ms}$ after stimulation. EMG for each muscle was processed by first removing the DC offset; then rectifying the signal. The latencies for withdrawal reflex onsets were designated when the reflex response exceeded a threshold of three standard deviations (SDs) above the mean EMG amplitude during a $100 \mathrm{~ms}$ pre-stimulus baseline period (-100msstimulation). Withdrawal reflex offsets were determined based on previous observations of voluntary withdrawal latencies, such that the response reported was limited to the nociceptive spinal pathways ${ }^{25}$. Withdrawal reflex responses in the upperlimb muscles were quantified by calculating the mean EMG for a time window between onset and offset latencies. The analyses were performed on each individual stimulation trial and the resulting values were averaged across the 8-stimuli for statistical comparisons. In addition, resultant two-dimensional (X-Y) endpoint force vectors were computed from the local peak force up to $350 \mathrm{~ms}$ for the 200 FLEX and EXT practice trials.

\section{Statistical analysis}

In the joint position task analysis of variance (ANOVA) was used to compare the direction and magnitude of the reflex endpoint force vectors across all arm positions. Arm position was considered as an independent factor and subject as a random factor for all multiple comparisons. Mean EMG reflex responses and onset latencies for each muscle were compared with separate ANOVAs across all positions. Bonferroni adjustments were made to correct for multiple comparisons in any additional post-hoc analysis. The forces, moments, EMG response amplitudes, and EMG onset latencies were compared between the initial and follow-up NEUT conditions, examining habituation, with independent $\mathrm{t}$-tests.

The conditioning task was examined using paired t-tests to compare the direction and magnitude of the endpoint force vectors, peak forces and moments, and mean EMG reflex responses and onset latencies before and after each set of practice trials. To enable comparisons between the EXT and FLEX conditions that were tested on different days the data was expressed as a percent change from pre-post practice $\left(i_{2}-i_{1} / i_{1}{ }^{*} 100\right)$ 
within each condition. The percent change in the direction and magnitude of the endpoint force vectors, peak forces and moments, and mean EMG reflex responses were then compared between EXT and FLEX conditions with independent t-tests. Onset latencies were compared between EXT and FLEX conditions as the absolute change from pre-post (ms). All data processing and statistical analyses were performed in MATLAB (Mathworks, MA, USA). Results were considered significant if $p<0.05$.

\section{Results}

\section{Joint position task}

The average withdrawal response evoked in the NEUT position for one subject is presented in Figure 2. Specifically, the withdrawal responses from all muscles except the erector spinae are displayed, along with the corresponding X-and Y-endpoint force traces. There were never any clear responses in the erector spinae, so that data is not presented. There were no differences in any of the EMG or force variables between the initial and follow-up NEUT position testing, suggesting there was minimal habituation to the stimulus, or at the least that the stimulation and recording conditions did not change. Onset latencies for the withdrawal responses were not significantly different across the arm positions for any of the muscles recorded. In addition, the amplitude of the withdrawal EMG response was not different across positions for any of the muscles shown in the grouped data.
The individual endpoint forces ( $\mathrm{Fx}, \mathrm{Fy})$ and moments $(\mathrm{Mx}, \mathrm{My})$ were not different across positions. When the endpoint force vectors were calculated from the peak Fx (+x) and Fy $(-y)$ magnitudes (posterior-lateral direction, see Figure 3), significant differences were present in the direction of withdrawal between the $\mathrm{SHL}_{\mathrm{FLEX}}$ and $\mathrm{SHL}_{\mathrm{EXT}}$ positions $(p<0.05)$. In addition, the magnitude of the endpoint force vector was significantly greater in the $\mathrm{ELB}_{\mathrm{EXT}}$ position than in the $\mathrm{ELB}_{\mathrm{FLEX}}$ or $\mathrm{SHL}_{\mathrm{FLEX}}$ positions $(p=0.025)$.

\section{Conditioning task}

The average withdrawal reflex response before and after EXT and FLEX practice is shown for the PD muscle and the endpoint force vectors for one subject in Figure 4. The only significant group difference observed in reflex onset latency after EXT practice was in the PD muscle where the response was observed at $78 \pm 11 \mathrm{~ms}$ before practice and $84 \pm 18 \mathrm{~ms}$ after EXT practice $(p=0.001)$. In the FLEX condition the reflex onset latency was significantly delayed after practice in the BRD ( $86 \pm 12 \mathrm{~ms}-94 \pm 18 \mathrm{~ms}, p=0.012)$, but no significant differences were noted for any other muscle in the FLEX condition. Furthermore, there were no significant differences in the pre-post absolute change in reflex latency (ms) between the EXT and FLEX conditions. Table 1 displays the variability in onset latency changes pre-post practice for each of the conditions. In addition, no differences were present in the EMG reflex response amplitudes for any of the muscles before and

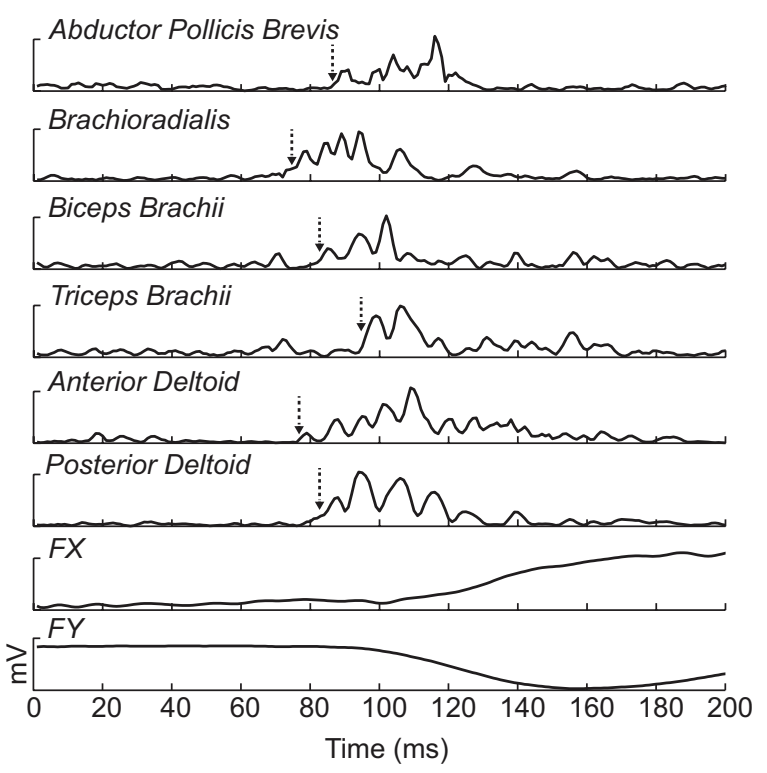

Figure 2. Representative data from one subject in the NEUT position where each trace represents the average of 8 individual stimulation responses. The average onset time is indicated by the dashed line with the arrow. The DC offset was removed and the signals were rectified prior to averaging for each channel. The time window is from the stimulation onset-200ms. 
A.

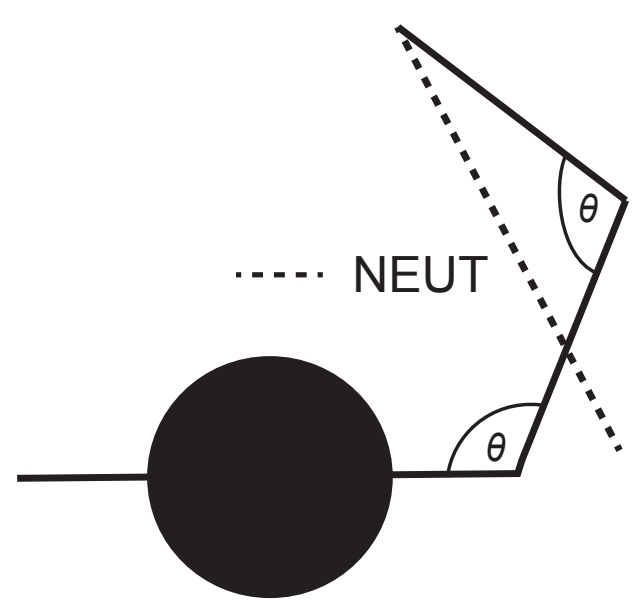

C.

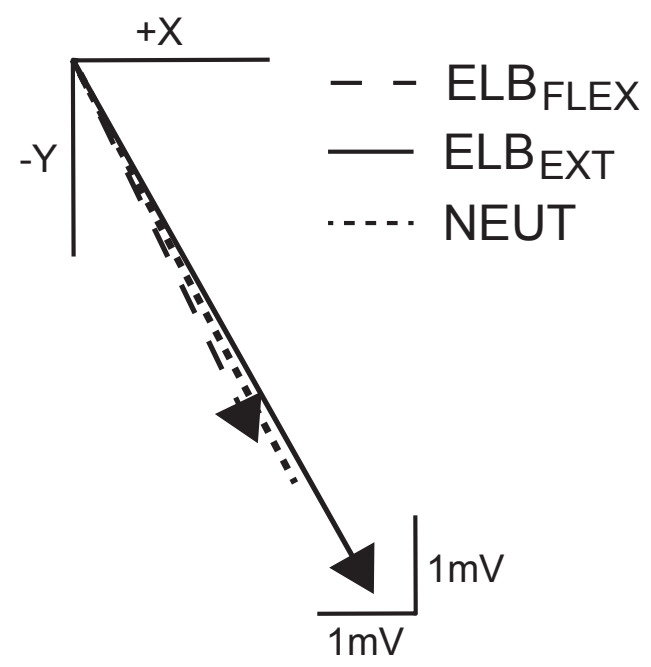

B.

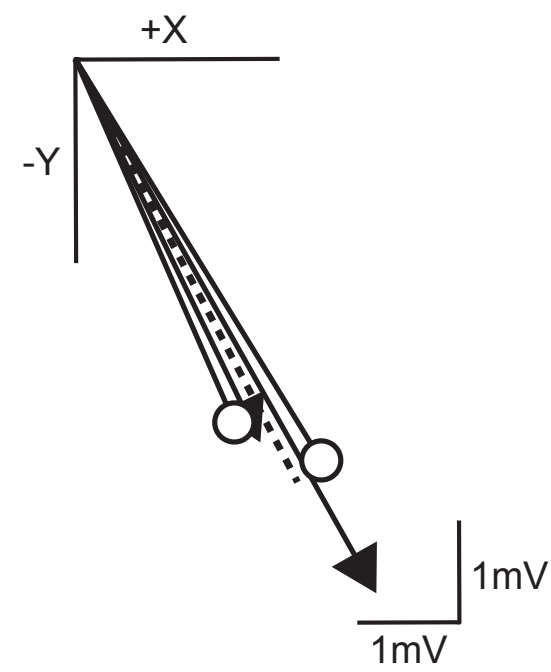

D.

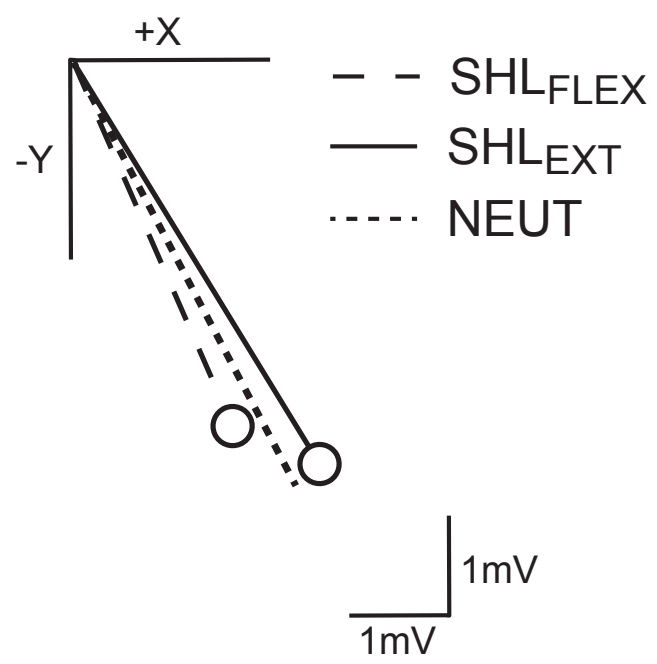

Figure 3. Results from the endpoint force vectors. A) Representative figure showing the arm in the NEUT position and the corresponding group force vector response to stimulation. B) Average force vector for all subjects in each position. The dashed line is the NEUT position. The lines with arrows are for the independent manipulation of the elbow angle, while the lines with circles correspond to changing shoulder angle. The same data from panel $\mathbf{B}$ is displayed in panels $\mathbf{C}$ and $\mathbf{D}$, separated for the elbow and shoulder positions, respectively.

after practice, for either EXT or FLEX conditions. Similarly, there were no differences between the EXT and FLEX practice conditions, with the exception of the AD muscle that showed a significantly greater decrease in the EMG reflex response in the FLEX condition $(p=0.037)$.

The individual endpoint peak forces and moments were not significantly different before and after either EXT or FLEX practice with the exception of the My-moment (pitch), which was significantly lower after EXT practice $(p=0.007)$. There were no differences in the direction or magnitude of the endpoint force vectors calculated from the peak $\mathrm{Fx}(+\mathrm{x})$ and Fy (-y) magnitudes (posterior-lateral direction) pre-post practice within either condition, or between EXT and FLEX conditions (Figure 5).

Electromyography and force recordings of nociceptive withdrawl responses during both joint manipulation and conditioning tasks.

160 Data Files

http://dx.doi.org/10.6084/m9.figshare.726964 
A.

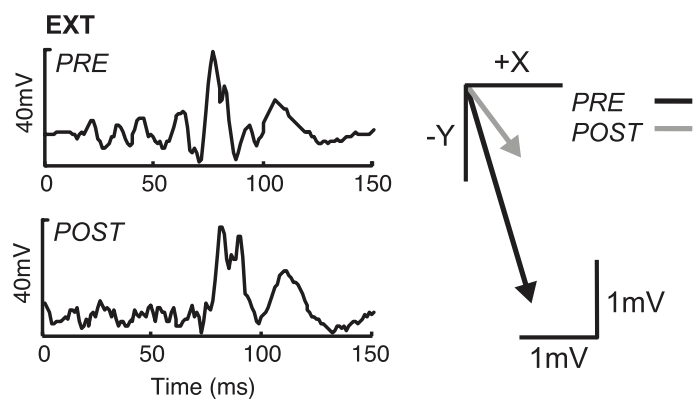

B.

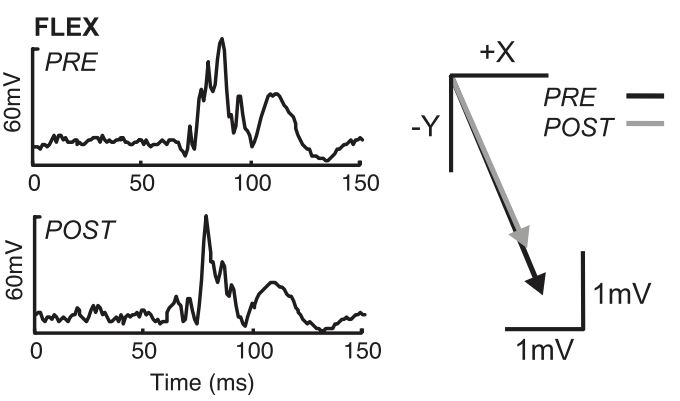

Figure 4. Representative nociceptive withdrawal responses before and after practice from the posterior deltoid (PD) muscle of one subject as well as the average direction and magnitude of the same subjects endpoint force vector for both A) EXT and B) FLEX practice.

Table 1. Mean change (+ increase, - decrease) of onset latencies from the electromyography (EMG) response after EXT and FLEX practice for each muscle and all subjects. The muscles listed are the biceps brachii long head (BBL), triceps brachii (TRI), brachioradialis (BRD), posterior deltoid (PD), anterior deltoid (AD), abductor pollicis brevis (APB), right erector spinae (ESR), and left erector spinae (ESL)

\begin{tabular}{|c|c|c|c|c|c|c|c|c|c|c|c|c|c|c|c|c|c|c|c|c|}
\hline \multirow[b]{3}{*}{$\mathrm{BBL}$} & \multicolumn{2}{|c|}{ Subject 1} & \multirow{2}{*}{\multicolumn{2}{|c|}{ Subject 2}} & \multirow{2}{*}{\multicolumn{2}{|c|}{ Subject 3}} & \multirow{2}{*}{\multicolumn{2}{|c|}{ Subject 4}} & \multirow{2}{*}{\multicolumn{2}{|c|}{ Subject 5}} & \multirow{2}{*}{\multicolumn{2}{|c|}{ Subject 6}} & \multirow{2}{*}{\multicolumn{2}{|c|}{ Subject 7}} & \multirow{2}{*}{\multicolumn{2}{|c|}{ Subject 8}} & \multirow{2}{*}{\multicolumn{2}{|c|}{ Subject 9}} & \multirow{2}{*}{\multicolumn{2}{|c|}{ Subject 10}} \\
\hline & EXT & FLEX & & & & & & & & & & & & & & & & & & \\
\hline & + & + & + & + & - & + & - & + & - & + & - & + & - & - & + & + & + & - & + & - \\
\hline TRI & + & - & + & + & + & + & - & + & - & - & + & - & - & - & - & - & + & + & + & - \\
\hline BRD & - & + & + & + & + & + & + & + & - & - & + & - & - & - & - & + & + & - & - & + \\
\hline PD & - & - & + & + & + & + & - & + & - & + & - & - & + & + & + & + & + & + & + & - \\
\hline$A D$ & + & + & - & + & + & + & + & - & - & + & - & - & - & + & + & + & - & + & - & + \\
\hline APB & + & + & + & + & + & - & + & + & + & + & - & - & + & + & - & + & - & - & - & + \\
\hline ESR & + & - & + & + & + & + & - & - & - & - & + & + & + & - & - & + & + & - & + & + \\
\hline ESL & + & - & + & - & - & - & + & - & - & - & - & + & + & - & + & - & - & - & - & - \\
\hline
\end{tabular}

\section{Discussion}

The purpose of the present study was to examine the adaptability of the nociceptive withdrawal reflex across different tasks. First, changing the elbow or shoulder joint angles independently resulted in a consistent nociceptive withdrawal response to the stimulation of the same receptive field on digit II. This conclusion is based on the pattern of withdrawal reflex activity in the upper-limb muscles being similar, regardless of the position of the limb. Some statistical differences were observed in the withdrawal force vectors, however, these differences did not correspond to changes in withdrawal reflex muscle activity across positions, suggesting they were due to altered limb biomechanics. More importantly, the direction of the withdrawal force response was relatively constant no matter the configuration of the upper-limb (see Figure 3). This was even the case when re-testing the NEUT position at the end of the experiment, where adjustments in limb position and repeated noxious stimuli did not show habituation in the nociceptive reflex, which has been observed previously ${ }^{2}$. Second, the nociceptive withdrawal response was resistant to change, at least to the short-term motor practice performed. While there were a few significant differences observed in 
A.

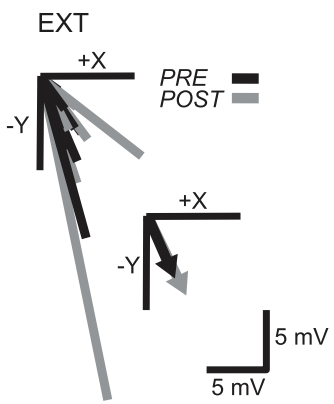

B.

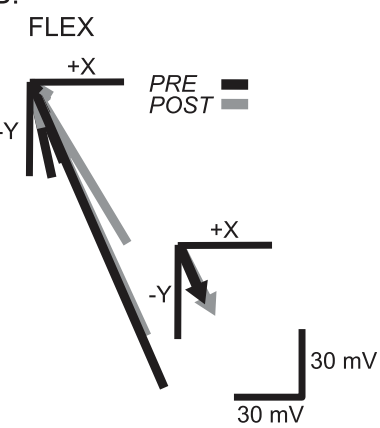

Figure 5. Group endpoint force vectors from the Fx (+x) and Fy (-y) before (black) and after (grey) practice for each subject with the A) EXT practice, and the B) FLEX practice. The inset on each panel shows the average for all subjects. No significant differences were noted.

individual EMG and force variables, the main observation was that the reflex responses were not consistently modified by either EXT or FLEX practice. If the nociceptive withdrawal reflexes in the upper limb are considered merely a protective mechanism then it would be expected that the response would be robust. These results lead us to conclude that the actionbased shaping of nociceptive reflex modules in the spinal cord help to prevent erroneous adaptations that would potentially compromise safety.

Much of the work examining the organization of nociceptive withdrawal reflexes has demonstrated distinct excitatory and inhibitory nociceptive reflex responses depending on the receptive fields stimulated (see review by Clarke and Harris $\left.(2004)^{6}\right)$. In the present study the withdrawal reflexes only elicited consistent excitatory responses in the upper-limb muscles regardless of the joint angle that was altered. This result is incongruent with the notion that the synergist muscles removing the limb should be excited while the opposing muscles should be inhibited ${ }^{3}$. It is difficult to ascertain the benefit of co-activating muscles throughout the entire upper limb in response to noxious stimulation, particularly if it slows down the physical withdrawal of the limb. Co-activation of the muscles throughout the upper limb in response to nociceptive stimulation could be specific to the spinal motor neurons being relatively quiescent during these trials, as reported previously ${ }^{26}$. There is also the potential that EMG cross-talk between opposing muscles (e.g. biceps brachii and triceps brachii) could have obscured smaller changes such as suppression, in particular with the muscle already quiescent ${ }^{27}$. Alternatively, there are distinct excitatory and inhibitory responses in upper limb muscles during movement, even demonstrating dependence on the phase of movement ${ }^{26,28}$. It has been suggested that tactile afferent input is organized by the actions the system performs routinely; for example, reaching and withdrawing the limb voluntarily ${ }^{10}$. If this nociceptive organization is linked to development $t^{13}$, or is action-based, it can be reasoned that the nociceptive withdrawal response at rest would be much less finely-tuned or that the synaptic input would be largely dispersed in the spinal cord. Specific to the present study, this rationale would also explain the similar directions of withdrawal for each of the arm configurations.

The original hypothesis that the nociceptive withdrawal reflex response would vary with the joint position was based on the expectation that each joint position would have an optimal withdrawal motor response and that the static muscle length changes around the elbow or shoulder joints would alter group II afferent input to interneurons that help to gate the withdrawal response $e^{20,21}$. It appears this assumption was too generalized since it is known that group II afferents from secondary muscle spindle endings have a relatively constant discharge rate and low discharge rate variability in static postures ${ }^{29-31}$; and thus the difference in group II afferent feedback may not have been sufficient to modulate nociceptive input. It has been suggested that the nociceptive withdrawal response arises from the integration of multiple sensory feedback sources, and that there is a complex sensorimotor transformation mediating the appropriate motor response $e^{8,10,15}$. In this case the group II afferent feedback alone would just be part of a larger, more complex set of sensory inputs that might be weighted differently across individual joints and arm positions. This would be feasible since there is a large amount of redundancy in multi-joint control of the upper limb and it has been reported that weighted sensory feedback in the upper limb improves control of the limb ${ }^{32}$. The five positions examined in the present study only encompassed a small area of the workspace of the upper limb, even confined to a single plane, so in this context very little weighting of sensory feedback would even be required to produce the same withdrawal response.

Nociceptive input is widely distributed to motorneurons innervating muscles across several joints and with different primary movements ${ }^{8}$, and because of this it was originally hypothesized that short-term motor practice would modulate the withdrawal reflex response. However, the results of the present study suggest that the motor practice the subjects performed was unable 
to alter this distribution of input. Presumably, this was because the practice was non-specific to the nociceptive withdrawal reflex, meaning that the subjects were practicing a voluntary motor task. However, the results of this study have caused us to re-examine the actual benefit of short-term plasticity in the nociceptive withdrawal reflex. Assuming the withdrawal reflex is organized with reflex modules, it is necessary for the reflex modules encoding nociceptive input to be developed over time and based on the motor actions commonly performed by the individual $^{37,8,10,14,15,33,34}$. This being the case, short-term adaptations would likely compromise the built-in protective capabilities of the nociceptive system. This is not to suggest that habituation and dishabituation (wind-up) of the nociceptive pain pathways does not occur ${ }^{2,16-19}$, but rather that the musculotopic organization underlying the activation of specific reflex modules ${ }^{9}$, ultimately producing the motor action, is relatively insensitive to short-term practice. This remains in agreement with the demonstrated results, however the task utilized in the current study may fail to elicit plasticity within the withdrawal reflex.

The results presented suggest potential environmental factors that must be taken into account. The timeline for the purported action-based shaping of reflex modules remains unclear. As mentioned previously, the model of action-based sensory encoding or somatosensory imprinting has been described in early post-natal development ${ }^{13,15}$, where spinal organization can be determined by simple tactile feedback from spontaneous muscle twitches during sleep ${ }^{35,36}$. However, the mature nociceptive system is much less flexible, presumably because withdrawal receptive fields have already been optimized to eliminate or depress erroneous connections and strengthen appropriate connections ${ }^{11,12}$. Consequently, to override, or adapt the adult nociceptive reflex modules to new biomechanical, anatomical, or action-based constraints there would need to be a much greater amount of practice. It has been suggested that the long-term plasticity occurs as a result of a greater transfer of information between primary motor cortex (M1)-basal ganglia-cerebellum ${ }^{37}$. Alternatively, the initial phase of adaptation can occur in as little as 10-30 minutes of practice, and can be driven by the disfacilitation of intracortical circuits in $\mathrm{M}^{38,39}$. Consequently, the short-term motor practice in the current study could have been specifically targeting the primary motor cortex and was not sufficient to cause adaptations in cerebellar circuits and pathways mediated through the cerebellum.

The original purpose of the present study was to examine the adaptability of the nociceptive withdrawal reflex across different tasks. The manipulation of joint position and reflex conditioning would activate specific reflex modules that would result in an efficient, coordinated limb withdrawal distinctly different from the control. The present results do not directly support the notion that different reflex modules were activated by independently changing elbow or shoulder joint angles. However, this does not suggest that there is no modular organization in the spinal cord for nociceptive input; rather it could just provide additional evidence that the reflex modules are specific to the receptive field activated. Instead, we hypothesize that the current data implies there is an additional layer of complexity to the sensorimotor transformation of nociception, meaning that the convergence of weighted multisensory input in the spinal cord was integrated to activate the same reflex module(s) for each arm position. In addition, since there appears to be a specific reflex encoder for each muscle in the dorsal horn of the spinal cord ${ }^{34}$, the integration of multisensory input had to be similar for each muscle to result in the same pattern of activation. Chang et al. ${ }^{40}$ recently demonstrated that whole limb kinematics are preferentially preserved over individual joint kinematics following peripheral nerve injury in the hindlimb of cats, and a similar compensatory mechanism may be responsible for the preservation of the withdrawal force direction despite altering individual joint angles. Thus, while there appears to be very complex processing of nociceptive input through interneuronal spinal circuits, the limb is consistently withdrawn in a general posterior direction towards the body. This could be a natural consequence of development ${ }^{15,35}$, or could be specific to non weight-bearing muscles that receive fewer cross-spinal inputs. Though it has been demonstrated repeatedly that the nociceptive withdrawal system is not hardwired, as was first believed ${ }^{5}$, the results of the present study imply that the nociceptive reflex modules are also resistant to short-term adaptations from practicing motor actions. This is consistent with the protective function of the nociceptive withdrawal reflex, as it guards the body from erroneous responses that could cause further harm. The data collected in the current study demonstrates a level of complexity within the nociceptive withdrawal reflex that was previously unclear. The results suggest that the motor response of the nociceptive withdrawal reflex remains fixed, which is in agreement with the long-term development of nociceptive reflexes, and regardless of the specific mechanisms involved, the nociceptive withdrawal response in the upper limb serves as an efficient means of protecting the hand.

\section{Author contributions}

Both authors were equally involved in the experimental design, data collection, data analysis, manuscript preparation, and manuscript revisions.

\section{Competing interests}

No competing interests were disclosed.

\section{Grant information}

The author(s) declared that no grants were involved in supporting this work. 
1. Dewald JP, Beer RF, Given JD, et al: Reorganization of flexion reflexes in the upper extremity of hemiparetic subjects. Muscle Nerve. (1999); 22: 1209-1221.

2. Floeter MK, Gerloff C, Kouri J, et al: Cutaneous withdrawal reflexes of the upper extremity. Muscle Nerve. (1998); 21: 591-598.

3. Schouenborg J, Kalliomaki J: Functional organization of the nociceptive withdrawal reflexes. I. Activation of hindlimb muscles in the rat. Exp Brain Res. (1990); 83: 67-78.

4. Schomburg ED, Steffens H, Marx B: Nociceptive reflexes evoked by TTX-resistant $\mathrm{C}$-fibre afferents and their sensitivity to opioids in the cat. Acta Physiol Pharmacol Bulg. (2001); 26: 193-196.

5. Eccles JC, Lundberg A: Synaptic actions in motoneurones by afferents which may evoke the flexion reflex. Arch Ital Biol. (1959); 97: 199-221.

6. Clarke RW, Harris J: The organization of motor responses to noxious stimuli. Brain Res Brain Res Rev. (2004); 46: 163-172.

7. Schouenborg J, Holmberg H, Weng HR: Functional organization of the nociceptive withdrawal reflexes. II. Changes of excitability and receptive fields after spinalization in the rat. Exp Brain Res. (1992) 90: 469-478.

8. Schouenborg J: Modular organisation and spinal somatosensory imprinting. Brain Res Brain Res Rev. (2002); 40: 80-91.

9. Levinsson A, Holmberg $\mathrm{H}, \mathrm{Broman} \mathrm{J}$, et al: Spinal sensorimotor transformation: relation between cutaneous somatotopy and a reflex network. J Neurosci. (2002); 22: 8170-8182.

10. Schouenborg J: Action-based sensory encoding in spinal sensorimotor circuits. Brain Res Rev. (2008); 57: 111-117.

11. Holmberg H, Schouenborg J: Developmental adaptation of withdrawal reflexes to early alteration of peripheral innervation in the rat. J Physiol. (1996); 495 (Pt 2): 399-409.

12. Holmberg H, Schouenborg J, Yu YB, et al: Developmental adaptation of rat nociceptive withdrawal reflexes after neonatal tendon transfer. J Neurosci. (1997); 17: 2071-2078.

13. Levinsson A, Luo XL, Holmberg $\mathrm{H}$, et al: Developmental tuning in a spinal nociceptive system: effects of neonatal spinalization. $J$ Neurosci. (1999); 19: 10397-10403.

14. Schouenborg J: Learning in sensorimotor circuits. Curr Opin Neurobiol. (2004); 14: 693-697.

15. Waldenstrom A, Thelin J, Thimansson E, et al: Developmental learning in a pain-related system: evidence for a cross-modality mechanism. J Neurosci. (2003); 23: 7719-7725.

16. Arendt-Nielsen L, Brennum J, Sindrup S, et al: Electrophysiological and psychophysical quantification of temporal summation in the human nociceptive system. Eur J Appl Physiol Occup Physiol. (1994); 68: 266-273.

17. Arendt-Nielsen L, Sonnenborg FA, Andersen OK: Facilitation of the withdrawal reflex by repeated transcutaneous electrical stimulation: an experimental study on central integration in humans. Eur J Appl Physiol. (2000); 81: 165-173.

18. Hagbarth KE: Spinal withdrawal reflexes in the human lower limbs. J Neurol Neurosurg Psychiatry. (1960); 23: 222-227.

19. Klein T, Magerl W, Hopf HC, et al: Perceptual correlates of nociceptive long-term potentiation and long-term depression in humans. J Neurosci. (2004); 24: 964-971.

20. Alstermark B, Lundberg A, Pinter M, et al: Subpopulations and functions of long C3-C5 propriospinal neurones. Brain Res. (1987); 404: 395-400

21. Alstermark B, Lundberg A, Pinter M, et al: Long C3-C5 propriospinal neurones in the cat. Brain Res. (1987); 404: 382-388.

22. Crowe A, Matthews PB: Further studies of static and dynamic fusimotor fibres. J Physiol. (1964): 174: 132-151.

23. Kofler M: Functional organization of exteroceptive inhibition following nociceptive electrical fingertip stimulation in humans. Clin Neurophysiol. (2003); 114: 973-980.

24. Kofler M, Stetkarova I, Wissel J: Nociceptive EMG suppression in triceps brachii muscle in humans. Clin Neurophysiol. (2004); 115: 1052-1056

25. Riley ZA, Krepkovich ET, Mayland EC, et al: Flexion-withdrawal reflexes in the upper-limb adapt to the position of the limb. Palo Alto, CA 2009.

26. Serrao M, Pierelli F, Don R, et al: Kinematic and electromyographic study of the nociceptive withdrawal reflex in the upper limbs during rest and movement. J Neurosci. (2006); 26: 3505-3513

27. Kofler M, Fuhr P, Leis AA, et al: Modulation of upper extremity motor evoked potentials by cutaneous afferents in humans. Clin Neurophysiol. (2001); 112: 1053-1063.

28. Don R, Pierelli F, Ranavolo A, et al: Modulation of spinal inhibitory reflex responses to cutaneous nociceptive stimuli during upper limb movement. Eur J Neurosci. (2008); 28: 559-568.

29. Burke D, Skuse NF, Stuart DG: The regularity of muscle spindle discharge in man. J Physiol. (1979); 291: 277-290.

30. Matthews PB: Evidence that the secondary as well as the primary endings of the muscle spindles may be responsible for the tonic stretch reflex of the decerebrate cat. J Physiol. (1969); 204: 365-393.

31. Matthews PB: A possible function for the secondary ending of the muscle spindle. J Physiol. (1969); 201: 102P-103P.

32. Bunderson NE, Ting LH, Burkholder TJ: Asymmetric interjoint feedback contributes to postural control of redundant multi-link systems. J Neural Eng. (2007); 4: 234-245.

33. Schouenborg J, Weng HR: Sensorimotor transformation in a spinal motor system. Exp Brain Res. (1994); 100: 170-174.

34. Schouenborg J, Weng HR, Kalliomaki J, et al: A survey of spinal dorsal horn neurones encoding the spatial organization of withdrawal reflexes in the rat. Exp Brain Res. (1995); 106: 19-27.

35. Petersson P, Granmo M, Schouenborg J: Properties of an adult spinal sensorimotor circuit shaped through early postnatal experience. J Neurophysiol. (2004); 92: 280-288.

36. Petersson $\mathrm{P}$, Waldenstrom A, Fahraeus $\mathrm{C}$, et al: Spontaneous muscle twitches during sleep guide spinal self-organization. Nature. (2003); 424: 72-75.

37. Pascual-Leone A, Nguyet D, Cohen LG, et al: Modulation of muscle responses evoked by transcranial magnetic stimulation during the acquisition of new fine motor skills. J Neurophysiol. (1995); 74: 1037-1045

38. Classen J, Liepert J, Wise SP, et al: Rapid plasticity of human cortical movement representation induced by practice. $J$ Neurophysiol. (1998); 79: 1117-1123.

39. Rosenkranz K, Kacar A, Rothwell JC: Differential modulation of motor cortical plasticity and excitability in early and late phases of human motor learning. J Neurosci. (2007); 27: 12058-12066.

40. Chang YH, Auyang AG, Scholz JP, et al: Whole limb kinematics are preferentially conserved over individual joint kinematics after peripheral nerve injury. J Exp Biol. (2009); 212: 3511-3521. 


\section{Open Peer Review}

\section{Current Peer Review Status:}

\section{Version 1}

Reviewer Report 10 December 2013

https://doi.org/10.5256/f1000research.1750.r1087

(C) 2013 Poston B. This is an open access peer review report distributed under the terms of the Creative Commons Attribution License, which permits unrestricted use, distribution, and reproduction in any medium, provided the original work is properly cited.

\section{Brach Poston}

Cleveland Clinic Lerner Research Institute, Cleveland, $\mathrm{OH}$, USA

The purpose of the present study was to examine the adaptability of the nociceptive withdrawal reflex in different task conditions after motor practice. Specifically, this was accomplished by applying electrical stimulation to the second digit in a total of five different arm positions (shoulder and elbow joint angle combinations), both before and after motor practice performed either in the opposite or same direction as the stereotyped withdrawal response.

The primary dependent measures of interest were the force responses recorded at the wrist and the EMG reflex responses obtained from six muscles of the upper limb. The main findings were:

1. The amplitude and onset latencies of the EMG reflex responses were similar in all of the arm configurations for every muscle studied.

2. There were minimal differences in the direction of the withdrawal force response for the different arm configurations.

3. A single session of motor practice in either the opposite or same direction as the stereotyped withdrawal response did not change the pattern of the EMG or Force responses.

The authors concluded that the nociceptive withdrawal response is resistant to short-term motor practice. Thus, if it is even possible to significantly modify the withdrawal reflex response, it would likely take extensive motor practice over a long period of time, which makes intuitive sense as it would not be beneficial for protective reflex responses to be modified by short-term perturbations. Therefore, it seems that the author's interpretation of their findings is appropriate.

Overall, the manuscript is very well-written, the data appear to have been collected carefully, and there are no major flaws or concerns, although the authors could probably have shortened the Introduction and Discussion sections.

The topic addressed seems to be relatively novel as I don't believe any previous studies in humans have addressed the degree to which the nociceptive withdrawal reflex can be modified in these task conditions with motor practice. Therefore, the current work makes a significant contribution 
to the current body of literature on the topic.

To the credit of the authors, they presented a balanced and justified interpretation of the results even though some of the findings did not match their original hypotheses. In addition, they pointed out any limitations of their study, which will have to be addressed in future research.

Finally, the focus of the research seems to be appropriate for F1000 Research and of interest to many readers of the journal.

Competing Interests: No competing interests were disclosed.

\section{I confirm that I have read this submission and believe that I have an appropriate level of expertise to confirm that it is of an acceptable scientific standard.}

Reviewer Report 02 December 2013

\section{https://doi.org/10.5256/f1000research.1750.r2598}

(C) $2013 \mathrm{Ge} \mathrm{H}$. This is an open access peer review report distributed under the terms of the Creative Commons Attribution License, which permits unrestricted use, distribution, and reproduction in any medium, provided the original work is properly cited.

\section{Hong-You Ge}

Center for Sensory-Motor Interaction, Aalborg University, Aalborg East, Denmark

The title of the article "Adaptability of the nociceptive withdrawal reflex" is not so specific to the current study. The title of the article should be confined to the main research results of the study, i.e., the nociceptive withdrawal reflex responses in the upper limb are resistant to the change of joint position and short term motor practice.

The abstract represents a suitable summary of the work. The experimental design and methods are appropriate and the discussion is well balanced. The conclusion is justified on the basis of the results of the study.

Competing Interests: No competing interests were disclosed.

I confirm that I have read this submission and believe that I have an appropriate level of expertise to confirm that it is of an acceptable scientific standard. 
The benefits of publishing with F1000Research:

- Your article is published within days, with no editorial bias

- You can publish traditional articles, null/negative results, case reports, data notes and more

- The peer review process is transparent and collaborative

- Your article is indexed in PubMed after passing peer review

- Dedicated customer support at every stage

For pre-submission enquiries, contact research@f1000.com 